\title{
Fractal Image Compression Based On Entropy Technique
}

\author{
JamilaH.Suad \\ Computer science, college of science/Al-Mustensiriyh University, Iraq
}

\begin{abstract}
Our proposed approach presents a method to reduce the encoding time called Fast Algorithm of Fractal Image Compression based on Entropy Values (FAFICEVs). This technique will be reducing the size of the domain pool. FFICEVs technique is based on the observation that many blocks domain are never used in a typical fractal encoding, and only a fraction of this large domain pool is actually used in the fractal coding. The collection of used domain blocks is localized in regions with high degree of structure. This technique focuses on the implementation issues and presents the first empirical experiments analyzing the performance of benefits of entropy approach to fractal image compression. The experiment is carried out with technique of quadtree partitioning, allowing up to three $(4 x 8,4 x 16$, and $8 x 16$ pixel) is examined. The new algorithm gives a good quality of reconstructed image with speeding in encoding time.
\end{abstract}

Keywords: entropy, fractal encoding, image compression, partitioning, and quadtree.

\section{Introduction}

Fractal image compression is a relatively recent image compression method which exploits similarities in different parts of the image. During more than two decades of development, the Iterated Function System (IFS) based compression algorithm stands out as the most promising direction for further research and improvement [1].

In the traditional fractal encoding, an image is divided into non-overlapping blocks called range blocks. For each range block, an exhaustive search is performed to find the optimal domain block which minimizes the mean square error (MSE), under certain affine contractive mapping. For each possible range block, the brute force domain-block search involves computing the optimal scaling factor and offset for each of the eight possible rotations and flipping is isometrics [2]. Therefore fractal encoding is the most consuming part in fractal image compression. One of the simplest ways to decrease encoding time of this full search problem is to decrease the size of the domain pool in order to decrease the number of domains to be searched [3].

There are regions of the image that are difficult to cover well using fixed size range blocks. Similarly, there are regions that could be covered well with larger size range blocks, thus reducing the total number of maps needed (and increasing the compression ratio). This observation leads to the use of the quadtree partitioning technique $[2,4]$. In a quadtree partition, range blocks as large as possible are used to code the image. When no good matching domain block (of one size bigger than the range block) can be found for a range block in the image, it is divided into four equally sized child range blocks [5].

In this paper we proposed a new technique to reduce the encoding time of fractal image compression by performing less searches as opposed to doing a faster search, by excluding many of domain blocks from the domain pool. This technique is based on the observation that many domains are never used in a typical fractal encoding, and only a fraction of this large domain pool is actually used in the fractal coding. The collection of used domains is localized in regions with high degree of structure.

\section{The Proposed New Technique}

The most computationally intensive part of the FIC process is the search step. One way to decrease encoding time is to decrease the number of comparisons between range and domain blocks. The proposed new technique called Fast Algorithm of Fractal Encoding based on Entropy Values (FAFEEVs) to reduce the domain pool of matching process between the range and domain block by using the value of entropy of range and domain blocks. Each range block is compared only with domain blocks having their entropy close to that of the range block. Indeed, for each range block $R_{i}$ we search a domain block $D_{j}$ such:

From (1), we can write:

$$
R_{i} \approx W_{i}\left(D_{j}\right)
$$

$$
\operatorname{entropy} R_{i} \approx \operatorname{entropy}\left(W_{i}\left(D_{j}\right)\right)
$$

The mapping $W_{i}$ is composed of two transformations: a scaling operation, multiplying all the pixel values in the block by a coefficient $s_{i}$ and an offset operation adding a constant $o_{i}$. We can write equation (2) such as: 


$$
W_{i}\left(D_{J}\right)=s_{i} D_{j}^{\sim}+o_{i}
$$

Where

$D^{\sim}$ is the mean of domain block.

Substituted equation (3) in equation (2) we obtained:

$$
\text { entropy } R_{i} \approx \operatorname{entropy}\left(s_{i} D_{j}^{\sim}+o_{i}\right)
$$

The translation coefficient (i.e. $o_{i}$ coefficient) did not change the entropy therefore equation (4) become:

$$
\operatorname{entropy} R_{i} \approx \operatorname{entropy}\left(s_{i} D_{j}^{\sim}\right) \text {....... }
$$

If we the left side of equation (5) subtracted from the right side of same equation then some error exist between them:

$$
\left|\operatorname{entropy}\left(R_{i}\right)-\operatorname{entropy}\left(s_{i} D_{j}^{\sim}\right)\right| \leq \varepsilon \ldots \ldots \ldots(6)
$$

All theoretical concepts tell us $\left|s_{i}\right|$ is always less than 1 (i.e. $\left|s_{i}\right|<1$ ) therefore equation (6) can be written as:

$$
\left|\operatorname{entropy}\left(R_{i}\right)-\operatorname{entropy}\left(D_{j}^{\sim}\right)\right| \leq \varepsilon
$$

Thus for every range block $R_{i}$, comparison is made only with domain blocks $D^{\sim}$ that satisfies the condition in equation (7). The value of parameter Edetermines the set of domain block that participate to the search of the best block that matches a given range block. If $\varepsilon$ is small, the encoding time is reduced but the image quality is diminished.

\section{The main steps of FAFEEVs can be summarized as:}

Step1: Load the image into buffer

Step2: Choose parameter $\varepsilon$

Step3: Partitioning the image into quadtree blocks size with non-overlap $\left(R_{l} \ldots R_{n}\right)$

Step4: Generate the domain image from the original image by averaging method

Step5: Build a new domain blocks $\left(D_{1}^{\prime} \ldots D_{m}^{\prime}\right)$

Step6: For each new domain block compute the entropy and for each range block compute the entropy

Step7: Compare between the range block and the domain block by using equation (4.7).

i. If the distance between entropy $\left(\mathrm{R}_{\mathrm{i}}\right)$ and entropy $\left(D_{m}^{\prime}\right)$ less than $\varepsilon$

ii. Then $D_{m}^{\prime}$ is the best matching:

- Compute the coefficient s

- Quantize the $s$;

- Compute $E\left(\operatorname{entropy}\left(R_{i}\right)\right.$, entropy $\left(D_{m}^{\sim}, s_{i}\right)$;

- If $E\left(\operatorname{entropy}\left(R_{i}\right)\right.$,entropy $\left(D_{m}^{\sim}, s_{i}\right)$ is minimum store the $I F S$-code; else go to the next domain block;

\section{Experimental Results and Discussion}

This section presents experimental results showing the efficiency of FAFEEVs. The performance tests carried out for a diverse set of well-known images of size 256x256 gray levels with 8bpp, coefficient (contrast) restricted to values between 0 and 1 in order to avoid searching domain pool twice (i.e. allowed only positive scaling factors in the gray level transformation). To ensure a compact encoding of the affine transformation, the value of contrast and brightness are quantized using 4 and 6 bits for contrast (scaling parameter) and brightness (offset parameter), respectively, hence thecompression ratio is $95 \%$ quadtree partitions. This study focuses on the implementation issues and presents the first empirical experiments analyzing the performance of benefits of entropy approach to fractal image compression. The experiment is carried out with technique of quadtree partitioning, allowing up to three $(4 \times 4,8 \times 8$, and $16 \times 16$ pixel) is examined. The result is shown in table (1) the encoding time scales linearly with the values of PSNR. The original Girl image of size $(256 \times 256)$ and the reconstructed Girl image used in our experiments (entropy, $\varepsilon=1.5$ ) and block size $4 \times 8$ are shown in Figure (1) and another example using original Bird image of size $(256 \times 256)$ and reconstructed image used (entropy, $\varepsilon=0.5$ ) and block size $4 \times 8$ shown in Figure (2) .In fact, the encoding time usually decrease whenever there is decreasing in the number of partitioning blocks. That's the aim of the new proposed technique, i.e. decreasing in encoding time with an accepted image. Table (2) also shows the effect of No. of partitioning blocks, then encoding time decrease since No. of partitioning blocks is directly proportional with PSNR. 
Table (1): The results of Entropy technique with Varity encoding parameters,applying on (A): Birds image, (B): Girl image.

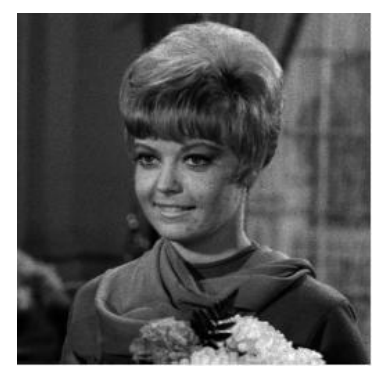

Original Image

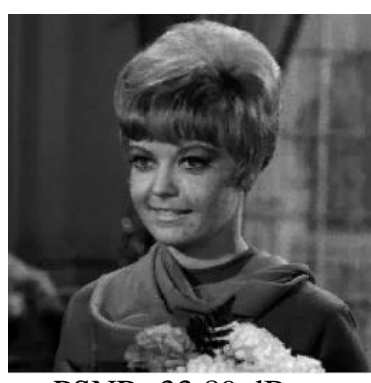

$\mathrm{PSNR}=33.89 \mathrm{~dB}$

$\mathrm{CR}=4.87$

$\mathrm{ET}=16 \mathrm{sec}$

Figure (1): The reconstructed image by using block size $=4 \mathrm{x} 8$ and entropy, $\varepsilon=1.5$.

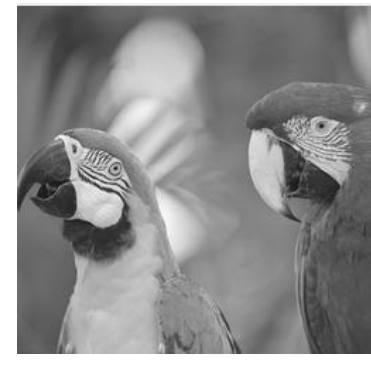

Original Image

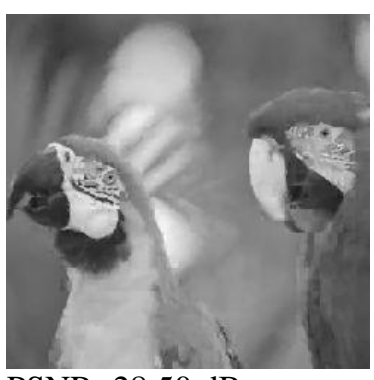

$\mathrm{PSNR}=28.50 \mathrm{~dB}$

$\mathrm{CR}=11.82$

$\mathrm{ET}=11 \mathrm{sec}$

Figure (2): The reconstructed image by using block size $=4 \times 8$ and entropy, $\varepsilon=0.5$.

Table (2): Show the relationship between encoding times with no. of partitioning blocks applying on (A): Birds image,

Girl

\begin{tabular}{|c|c|c|c|c|l|l|l|}
\hline & \multirow{2}{*}{$\begin{array}{c}\text { Bloc } \\
\mathbf{k}\end{array}$} & \multicolumn{3}{|c|}{ Birds Image } & \multicolumn{3}{c|}{ Girl Image } \\
\cline { 3 - 8 } & $\begin{array}{l}\text { Size } \\
\text { No. of } \\
\text { blocks }\end{array}$ & $\begin{array}{l}\text { PSNR } \\
\text { (dB) }\end{array}$ & $\begin{array}{l}\text { ET } \\
(\mathbf{s e c})\end{array}$ & $\begin{array}{l}\text { No. } \\
\text { blocks }\end{array}$ & $\begin{array}{l}\text { PSNR } \\
\text { (dB) }\end{array}$ & ET(sec) \\
\hline 0.5 & $4 \times 8$ & 2398 & 30.15 & 16 & 2911 & 34.20 & 18 \\
\hline 1 & $4 \times 8$ & 1198 & 29.85 & 15 & 1200 & 33.89 & 16 \\
\hline 1.5 & $4 \times 8$ & 1081 & 28.50 & 12 & 1165 & 33.86 & 14 \\
\hline 2 & $4 \times 8$ & 995 & 27.91 & 11 & 1026 & 33.59 & 12 \\
\hline 0.5 & $4 \times 16$ & 1084 & 20.29 & 15 & 1756 & 32.23 & 16 \\
\hline 1 & $4 \times 16$ & 994 & 20.15 & 14 & 1543 & 31.71 & 14 \\
\hline 1.5 & $4 \times 16$ & 893 & 20.12 & 13 & 993 & 31.45 & 13 \\
\hline 2 & $4 \times 16$ & 773 & 19.92 & 12 & 876 & 30.52 & 11 \\
\hline 0.5 & $8 \times 16$ & 856 & 20.01 & 14 & 975 & 28.14 & 15 \\
\hline 1 & $16 \times 8$ & 721 & 19.45 & 13 & 834 & 28.08 & 14 \\
\hline 1.5 & $8 \times 16$ & 654 & 18.12 & 21 & 733 & 27.45 & 12 \\
\hline 2 & $8 \times 16$ & 549 & 17.87 & 10 & 602 & 26.37 & 11 \\
\hline
\end{tabular}

(B):

image. 


\section{Conclusions}

We can see in the above tables and figures, differententropy values with other fractal encode parameters are kept (i.e., number of quantized scale and offset, error value, mean and standard deviation)different values for block numbers, compression ratio and PSNR can be achieved. But we have found that the FAFEEVs technique no need to symmetry states to find the best matching between range blocks and domain blocks. And about the same reconstructed image quality can be achieved in FAFEEVs technique with less number of blocks than in traditional FIC technique. This means that we can gate a high reconstructed image quality with more compression ratio using FAFEEVs technique than using traditional techniques.

\section{References}

[1] M. Barnsley and L. P. Hurd, Fractal Image Compression (AK Peters.Ltd, 1993).

[2] Fisher Y., Fractal Image Compression: Theory and Application (SpingerVerlag Edition, New York, 1995).

[3] Sofia Doudaet. al., A new approach for improvement of fractal image encoding ,International Journal on Computer Science and Engineering ,2(4),2010, 1387-1394.

[4] CorinaS_araru, Image Compression Based on Fractal Properties: Fractal Image Compression Optimization Methods, Nonlinear Phenomena in Complex Systems, 11 (2), 2008, 184 - 186

[5]. Jamila H., Fractal Image compression, doctoral thesis, college of science, university of Baghdad,Iraq,2001. 